\title{
International Journal of Sports Medicine - Ethical Standards in Sport and Exercise Science Research
}

D. J. Harriss

G. Atkinson

Bibliography

DOI $10.1055 / \mathrm{s}-0029-1237378$

Int J Sports Med 2009; 30:

701-702 @ Georg Thieme

Verlag KG Stuttgart - New York

ISSN 0172-4622

\section{Correspondence}

\section{J. Harriss}

Research Institute for Sport and Exercise Sciences

Henry Cotton Campus

Liverpool John Moores

University

Webster Street

Liverpool L3 2ET

United Kingdom

Tel.: +441512314427

Fax: +441512314353

D.Harriss@ljmu.ac.uk
For research that involves human participants or animals to be published in the International Journal of Sports Medicine (IJSM), it must have been conducted in accordance with recognised ethical standards and national/international laws. Authors are required to confirm that these standards and laws have been adhered to by formally citing this editorial within the methods section of their own manuscript. In this editorial, we synthesise the standards and laws into one source for convenience to authors of IJSM submissions. We outline the principles of the World Medical Association Declaration of Helsinki [1] and the Institute for Laboratory Animal Research of the National Research Council's Guide for the Care and Use of Laboratory Animals [2]. We highlight ethical issues included in national/international law and provide guidance on ethical issues common to Sport and Exercise Science.

Authors who cite this editorial confirm that research using human participants has been conducted ethically according to the principles of the Declaration of Helsinki. The Declaration is intended to be read as a whole and every principle is equally important, but those points mostcommonly considered by sport and exercise scientists are summarised below.

1. Basic principles. Respect the rights and welfare of participants which must take precedence over all other interests.

2. Ethical review. Before research begins and before amendments are applied, research must be reviewed and approved by an appropriate ethics committee.

3. The research protocol. The study, research design and statistical analysis must be clearly described, justifiable and appropriate. In drawing up the research protocol, the researcher must;

a) consider ethical issues in accordance with the Declaration of Helsinki,

b) provide information regarding funding, sponsors, institutional affiliations, other potential conflicts of interest,

c) consider the contribution to new knowledge and consider the environment,

d) include details of any incentives for participants and provisions for treating and/or compensating participants who are harmed as a consequence of participation in the research study,

e) describe the arrangements for post-study access by all participants to interventions identified as beneficial in the study or access to other appropriate care or benefits.
4. Consent. Informed consent/assent should be provided freely by the participant and should ideally be in writing. If written consent/assent cannot be obtained, or is not appropriate, then oral consent/assent should be formally documented and witnessed. Research that involves children or other populations that cannot consent (e.g. vulnerable populations) should seek consent from an appropriate person and assent from the participant. Research involving participants who are physically or mentally incapable of giving consent may be undertaken only if the physical or mental condition that prevents giving informed consent is a necessary characteristic of the research population. Informed consent/assent must include the;

a) aims of the research,

b) methods,

c) sources of funding,

d) conflicts of interest,

e) institutional affiliations,

f) anticipated benefits and potential risks,

g) potential discomfort and

h) right to refuse to participate or withdraw consent without reprisal.

5. Conduct. Research must be conducted;

a) in accordance with appropriate risk management

b) by appropriately qualified researchers and support staff

c) with skill and care

d) in an appropriate setting

e) in order to protect the privacy of participants and confidentiality of their personal information

f) in accordance with laws and regulations of the country or countries in which the research is to be performed as well as international norms and standards. Specific laws relevant to research ethical may regulate the collection, use and/or storage of human tissue; the protection of individuals that lack the capacity to consent; data protection; and the use of drugs in research.

6. Governance. Serious adverse events occurring during the study must be reported to the ethics committee that ethically reviewed and approved the research.

Authors who cite this editorial confirm that research involving animals has been conducted ethically according to the principles of the Guide for the Care and Use of Laboratory Animals of the Institute for Laboratory Animal Research [2]. Again, the guide is intended to be read as a whole, 
but the basic obligations on the researcher are summarised below. The researcher must;

1. Ensure the appropriateness of experimental methods.

2. Legally acquire animals.

3. Ensure that animals are properly housed and fed to ensure safe, hygienic and comfortable living conditions.

4. Maintain a record of animal care.

5. Ensure that animal maintenance and research are carried out by qualified personnel, following all legal statutes and regulations.

6. Administer appropriate pain management to minimize suffering, discomfort and pain.

Specific issues relevant to Sports Medicine:

1. Retrospective ethics. Data are routinely collected from individuals for various purposes. For example, sport scientists may monitor physiological function of an athlete in order for him or her to gain an edge over their rivals. Data collected exclusively for one purpose cannot be used for another purpose (research) unless consent for the use in research is subsequently given and the research ethically approved. An exception to this would be where the data collected for the primary purpose is anonymised prior to use in a research study (second purpose) which has ethical approval. Retrospective ethical approval cannot be granted for any research study.

2. The use of placebo. The inclusion of a placebo group in a research study may challenge the principle of equipoise. Ideally, participants should be randomly assigned to experimental or placebo groups. In healthy volunteers, where possible, the type of treatment should be blinded, for example, comparator (control) participants could be given a fitness or lifestyle information sheet. After a finite length of time those participants in the comparator group could be offered the experimental condition, or an experiment could be halted if at any point it became clear that the placebo group was fairing more poorly [4]. In more medical research where participants are patients or clients extreme care must be taken to avoid the abuse of placebo. In this type of research, the use of placebo is acceptable when no current proven intervention exists, or when the participant will not be at risk of serious or irreversible harm.

3. Deception. The use of deception in research (e.g. in a pacing strategy study in which time trial distance is deceived) must be merited such that there are no reasonable alternatives for obtaining the data, as long as there is no reasonable expectation to cause pain or severe emotional distress. If deception is to be used, then the participants must be accurately informed of the risks and be debriefed at the conclusion of the study with the option to withdraw their data [5].

4. Good research practice. The UK Medical Research Council has outlined some basic principles of good research practice which may help to ensure that research is conducted ethically. These include planning, conduct, recording data, reporting results, applying the results [3]. Relevant issues not specifically raised in the Declaration of Helsinki include the use, calibration and maintenance of equipment, $\mathrm{COSHH}$, documentation of standard operating procedures, retention of data, publication policy, authorship, correction of errors and retraction of published findings and intellectual property rights.

\section{References}

1 Declaration of Helsinki. World Medical Association. Available at http:// www.wma.net/e/ethics unit/helsinki.htm

2 Institute for Laboratory Animal Research, National Research Council. Guide for the Care and Use of Laboratory Animals. Washington, D.C.: National Academy Press; 1996; 140

3 Medical Research Council. MRC ethics series: Good research practice 2005. London: Medical Research Council; 2005; 1-16

4 Shephard RJ. Ethics in exercise science research. Sports Med 2002; 32: 169-183

5 The American Psychological Association. Ethical principles of psychologists and code of conduct. Am Psychol 2002; 57: 1060-1073 